\title{
Mathematical model of dust collection process in apparatus with counter-swirling flows in aspiration systems
}

\author{
I.V. Stefanenko \\ Institute of Architecture and Civil Engineering Volgograd \\ State Technical University \\ Department of Building Materials and Special Technologies \\ Volgograd, Russia.
}

T.V. Solovyova

Associate Professor of Volgograd State Technical University (Department of Mathematics and Information Technologies), Volgograd, Russia.

\author{
V.N. Azarov \\ Volgograd State Technical University \\ Department of Life Safety in Construction and Urban \\ Management \\ Volgograd, Russia. \\ T.A.Kislenko \\ Volga territorial department of the Federal Service for \\ Environmental, \\ Technological and Nuclear Supervision of Rostekhnadzor \\ Volgograd, Russia.
}

A. M. Redhwan

Volgograd State University of Architecture and Civil Engineering Department of "Life Safety in Technosphere"

Volgograd, Russia awadhredhwan_87@mail.ru

\begin{abstract}
The article substantiates the importance of environmental safety and labor protection in industrial enterprises; special attention is paid to the content of fine dust. The problems of trapping fine dust particles in a centrifugal field with the help of vortex dust collectors of CSF are considered. To describe the processes of dust collection, the authors selected a probabilistic-stochastic approach that makes it possible to make the transition from the probability of elementary and group events in the process of mass transfer of dust particles to the efficiency of dust separation. A method is presented for determining the probability of mass transfer of dust particles in apparatus with counter-swirling flows. The results of probability calculations for the lower input of the CSF apparatus are presented, depending on various parameters.
\end{abstract}

Keywords-vortex technologies, centrifugal field, dust separators, Markov process, dust mass, fractionation efficiency of dust collection, Pontryagin-Boguslavsky equation.

\section{INTRODUCTION}

In many cases, dust-producing zones from different sources intersect with working zones on production sites of industrial enterprises (enterprises producing asbestos-cement, asphalt-concrete, etc.). At the same time, it is necessary to address the tasks of environmental safety and labor protection [1-5].
The trapping of fine dust particles in a centrifugal field is one of the most important areas in which vortex technologies are used. To solve such problems, dust collectors with counter swirling flows (CSF) are used. Their constructive feature is that the secondary flow curler is moved out of the body of the apparatus. This type of dust collector requires precise control and monitoring of the operating mode of the apparatus. This is due to the fact that the flow pattern of the lower inlet pipe must be preserved, which has a twisted character at the entrance to the separation chamber. Since there are reverse current zones in the swirling current, the most favorable dust collection mode is created while maintaining the necessary intensity of the swirling of the dust flow after the tangential twist and before entering the separation chamber. This mode of operation of the CSF apparatus makes it possible to achieve the lowest energy use (Figure1). In dust collecting apparatuses, as seen in the diagram, the mass transfer of dust particles flows in the upper zone $\mathrm{A}$ and the lower zone B simultaneously. The flows curl in one direction, but towards each other. The processes of mass transfer in the centrifugal field in each of the zones are described taking into account deterministic and stochastic phenomena. Deterministic regularities are determined by the fundamental laws of physicochemical mechanics. Stochastic phenomena have a significant effect on the process in reality [6-9]. 


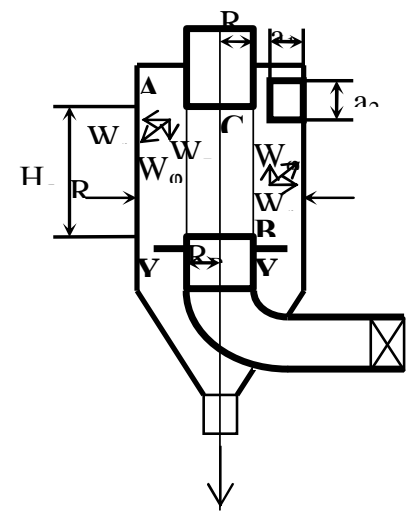

Fig. 1. Diagram of dust-gas flow motion in apparatuses of CSF

\section{METHOD FOR DESCRIBING THE PROCESSES OF DUST COLLECTION}

To describe these dust collection processes, the authors chose a probabilistic-stochastic approach. It shows clearly the transition from the probability of elementary and group events in the process of transfer to the efficiency of dust removal.

The CSF apparatuses are devices which efficiency depends on the design parameters, the operating mode, the volume of air coming from the hopper, the mass of the dust particles, the velocities and properties of the dust-gas flow [10-12].

The authors considered a continuous one-dimensional Markov process $\lambda(t)$ with fixed value $\lambda(0)=\lambda_{0}$ at the initial moment $t=0$, which is inside an interval $(c, d)$, i.e. the initial probability density is a delta function:

$$
\tilde{p}(\lambda, 0)=\tilde{p}_{0}(\lambda)=\delta\left(\lambda-\lambda_{0}\right), \quad \lambda_{0} \in(c, d) .
$$

A problem was posed in determining the probability that a random process with an initial value $\lambda_{0}$, within the time of $t>$ 0 will reach the boundaries of the interval $(c, d)$, i.e. either boundary $c$ or boundary $d$.

Pontryagin L.C. [13] showed that probability $p\left(t, \lambda_{0}\right)$ of reaching of the boundaries satisfies the differential equation:

$$
\frac{\partial p\left(t, \lambda_{0}\right)}{\partial t}=a\left(\lambda_{0}\right) \frac{\partial p\left(t, \lambda_{0}\right)}{\partial \lambda_{0}}+\frac{1}{2} b\left(\lambda_{0}\right) \frac{\partial^{2} p\left(t, \lambda_{0}\right)}{\partial \lambda_{0}^{2}}
$$

where $a\left(\lambda_{0}\right)$ and $b\left(\lambda_{0}\right)$ are the coefficients of drift and diffusion, when the initial (2) and boundary (3) conditions are met.

For all the values of $\lambda_{0} \in(c, d)$ the probability of reaching the boundaries at $t=0$ is zero:

$$
p\left(0, \lambda_{0}\right)=0, \quad c<\lambda_{0}<d
$$

At the boundaries of the interval, i.e. at $\lambda_{0}=c$ and $\lambda_{0}=d$, the probability equals one:

$$
p(t, c)=p(t, d)=1
$$

In addition to these conditions, the following relationship must be fulfilled:

$$
\lim _{t \rightarrow \infty} p\left(t, \lambda_{0}\right)=1, c \leq \lambda_{0} \leq d
$$

Additionally, probability $q\left(t, \lambda_{0}\right)=1-p\left(t, \lambda_{0}\right)$ needs to be determined as not reaching the boundary $c$ or $d$, i.e. probability of not being out of permissible range $(c, d)$.

$$
q\left(t, \lambda_{0}\right)=P(c<\lambda(t)<d ; 0<t<T), \lambda_{0} \in(c, d), \mathrm{T}=\mathrm{T}\left(c, \lambda_{0},\right.
$$

$d$ ) is a random moment of time of first reaching of boundary $c$ or $d$.

In this case, equation (1) is also valid, but the boundary conditions change:

$$
\begin{aligned}
& q\left(0, \lambda_{0}\right)=1, \quad c<\lambda_{0}<d ; \\
& q(t, c)=q(t, d)=0 .
\end{aligned}
$$

For a cylindrical problem applicable to vortex dust collectors, Boguslavsky E.I. [14] rewrote the equation (1) in polar coordinates $(r, \varphi)$. He proposed to find the probability of mass transfer of dust particles with a diameter $d$ from the equation, which was called the Pontryagin-Boguslavsky equation:

$$
\begin{aligned}
& \frac{\partial P_{A}}{\partial \tau}=W_{r} \frac{\partial P_{A}}{\partial r}+\frac{W_{\varphi}}{r} \frac{\partial P_{A}}{\partial \varphi}+0,5 b_{r} \frac{\partial^{2} P_{A}}{\partial r^{2}} \\
& \frac{\partial P_{B}}{\partial \tau}=W_{r} \frac{\partial P_{B}}{\partial r}+\frac{W_{\varphi}}{r} \frac{\partial P_{B}}{\partial \varphi}+0,5 b_{r} \frac{\partial^{2} P_{B}}{\partial r^{2}}
\end{aligned}
$$

where $P_{A}, P_{B}$ - the probabilities a dust particle transfer to the upper and lower inputs of the apparatus; $W_{r}, W_{\varphi}-$ velocities of particles of the dust-gas flow; $b_{r}$ - coefficient of particle diffusion along the axis $r$.

Under the guidance of Boguslavsky E.I., the solution to the equation was obtained by the method of separation of the equation by physical processes, which made it possible to reduce each of the initial equations to the solution of two. So for the bottom input $B$ we have:

$$
\begin{gathered}
\frac{\partial P_{1 B}}{\partial \tau}=0,5 b_{r} \frac{\partial^{2} P_{1 B}}{\partial r^{2}} \\
\frac{\partial P_{2 B}}{\partial \tau}=W_{r} \frac{\partial P_{2 B}}{\partial r}+\frac{W_{\varphi}}{r} \frac{\partial P_{2 B}}{\partial \varphi}
\end{gathered}
$$

Solutions for the equations (6) - (7) look as follows:

$$
\begin{gathered}
P_{1 B}=\operatorname{erf}(Y), \\
\operatorname{erf}(Y)=\frac{2}{\sqrt{\pi}} \int_{0}^{Y} e^{-t^{2}} d t \\
Y=\sqrt{\frac{R^{2}}{2 \mu^{2} b\left(\tau-\tau_{0}\right)}}
\end{gathered}
$$

where $R$ - apparatus radius; $\mu$ - coefficient of dynamic viscosity; $b$ - diffusion coefficient of dust particle; $\left(\tau-\tau_{0}\right)-$ particle time in the apparatus, 


$$
P_{2 B}=\left(1-c e^{-\lambda r_{H}\left(1-W_{r} / V_{r}\right)}\right)
$$

where $\lambda$ - variables separation parameter; $r_{H}$ - point of particle coordinate entering the apparatus; $W_{r}, V_{r}$ - radial velocities of particles and gas; $c$ - constant of integration.

When solving the equation (7) the following transformation was used:

$$
\varphi=\frac{W_{\varphi}}{r \cdot V_{r}}
$$

Thus, the fractional dust collection efficiency for particles with a diameter $d$ entering the apparatus at a point with the coordinate $r=r_{H}$, will be determined by the formula (8):

$$
P=\left(1-c e^{-\lambda r_{H}\left(1-W_{r} / V_{r}\right)}\right) \cdot \operatorname{erf}(Y)
$$

The probability of mass transfer of dust particles along the inlet branch of zone $B$ is:

$$
P_{B}=\frac{1}{R_{H}-R_{T}} \int_{R_{T}}^{R_{H}} P d r_{H}
$$

or

$$
P_{B}=\left(1-c \cdot \frac{e^{-\lambda E_{B} R_{T}}-e^{-\lambda E_{B} R_{H}}}{\lambda \cdot E_{B}\left(R_{H}-R_{T}\right)}\right) \cdot \operatorname{erf}(Y)
$$

where $R_{H}, R_{T}$ - radii of the cylindrical part of the body and the exhaust pipe; $E_{B}=1-W_{r} / V_{r}$.

The constant of integration $c$ is determined from the physically justified condition that $P_{B}=0$ at $W_{r}=V_{r}$.

Then $E_{B}=0$ and to determine that value of $c$ we have:

$$
1=c \cdot \frac{e^{-\lambda E_{B} R_{T}}-e^{-\lambda E_{B} R_{H}}}{\lambda E_{B}\left(R_{H}-R_{T}\right)}
$$

The limit is calculated by the rule of L'Hospital:

$$
\lim _{E_{B} \rightarrow 0} \frac{e^{-\lambda E_{B} R_{T}}-e^{-\lambda E_{B} R_{H}}}{\lambda E_{B}\left(R_{H}-R_{T}\right)}=\lim _{E_{B} \rightarrow 0} \frac{-\lambda R_{T} e^{-\lambda E_{B} R_{T}}+\lambda R_{H} e^{-\lambda E_{B} R_{H}}}{\lambda\left(R_{H}-R_{T}\right)}=\frac{-\lambda R_{T}+\lambda R_{H}}{\lambda\left(R_{H}-R_{T}\right)}=1
$$

Therefore, the constant of integration $c=1$.

Finally, for zone $B$ we obtain the probability of mass transfer:

$$
P_{B}=\left(1-\frac{e^{-\lambda E_{B} R_{T}}-e^{-\lambda E_{B} R_{H}}}{\lambda \cdot E_{B}\left(R_{H}-R_{T}\right)}\right) \cdot \operatorname{erf}(Y)
$$

The parameter of separation of variables $\lambda$ is determined from the boundary conditions $W_{r}=0$ at $P_{B}=0,5$, using formula (8)

We have:

$$
\begin{aligned}
& 0,5=\left(1-e^{-\lambda r_{H}}\right) \operatorname{erf}(Y), \\
& \frac{1}{2 \operatorname{erf}(Y)}=1-e^{-\lambda r_{H}} \quad \text { or } \quad e^{-\lambda r_{H}}=1-\frac{1}{2 \operatorname{erf}(Y)}
\end{aligned}
$$

Expression of determining parameter $\lambda$ is obtained using logarithm:

$$
\lambda=-\frac{1}{r_{H}} \ln \left(1-\frac{1}{2 \operatorname{erf}(Y)}\right)
$$

where

$$
Y=\sqrt{\frac{R^{2}}{2 \mu^{2} b\left(\tau-\tau_{0}\right)}}=k \cdot R=k \cdot\left(R_{H}-R_{T}\right)
$$

$k$ - empirical coefficient.

Formula (10) holds true for all $\operatorname{erf}(Y)>0,5$, and thus the value $k>1$, and for all $\quad k \geq 10 \quad \operatorname{erf}(Y)=1$.

\section{CONCLUSION}

The authors created a program for integrated mathematical system MathCAD for calculating integral $\operatorname{erf}(Y)$, the separation parameters for variables $\lambda$, the probability for lower input based on different values of $k, r_{H}$ и $E_{B}$ with the values of design parameters $R_{H} ; R_{T}$, that are typical for CSF $400 \mathrm{ml}$. The obtained results are summarized in Tables 1 and 2 .

TABLE I. PROBABILITY OF THE PB FOR TRAPPING PARTICLES ENTERING THE APPARATUS THROUGH THE LOWER INPUT AT $\mathrm{R}_{\mathrm{H}}=0,11 ; \mathrm{R}_{\mathrm{H}}=0,4 ; \mathrm{R}_{\mathrm{T}}=0,1$

\begin{tabular}{ccc||ccccc}
\hline \hline \multirow{2}{*}{$k$} & \multirow{2}{*}{$\operatorname{erf}(Y)$} & $\lambda$ & \multicolumn{5}{c}{ with $E_{B}$} \\
\cline { 4 - 8 } & & & 0,5 & 0,7 & 0,8 & 0,9 & 0,99 \\
\hline \hline 2 & 0,60 & 16,00 & 0,50 & 0,55 & 0,56 & 0,57 & 0,58 \\
3 & 0,80 & 8,98 & 0,52 & 0,61 & 0,64 & 0,66 & 0,68 \\
4 & 0,91 & 7,24 & 0,52 & 0,64 & 0,70 & 0,70 & 0,73 \\
5 & 0.97 & 6,63 & 0,53 & 0,64 & 0,68 & 0,72 & 0,75 \\
10 & 1,00 & 6,30 & 0,52 & 0,64 & 0,68 & 0,72 & 0,75 \\
\hline \hline
\end{tabular}

TABLE II. PROBABILITY OF THE PB FOR TRAPPING PARTICLES ENTERING THE APPARATUS THROUGH THE LOWER INPUT AT $\mathrm{R}_{H}$ $=0,02 ; \mathrm{R}_{\mathrm{H}}=0,4 ; \mathrm{R}_{\mathrm{T}}=0,1$

\begin{tabular}{lcc||ccccc}
\hline \hline \multirow{2}{*}{$k$} & $\operatorname{erf}(Y)$ & $\lambda$ & \multicolumn{5}{c}{ with $E_{B}$} \\
\cline { 4 - 8 } & & & 0,5 & 0,7 & 0,8 & 0,9 & 0,99 \\
\hline \hline 2 & 0,60 & 16,00 & 0,60 & 0,60 & 0,60 & 0,60 & 0,60 \\
3 & 0,80 & 8,98 & 0,79 & 0,80 & 0,80 & 0,80 & 0,80 \\
4 & 0,91 & 7,24 & 0,89 & 0,90 & 0,91 & 0,91 & 0,91 \\
5 & 0.97 & 6,63 & 0,94 & 0,96 & 0,96 & 0,96 & 0,96 \\
10 & 1,00 & 6,30 & 0,97 & 0,99 & 0,99 & 0,99 & 0,99 \\
\hline \hline
\end{tabular}

Comparison of the results shows that the dust collection efficiency for the lower input varies from 0,602 to 0,997 with $r_{H}=0,02$ depending on the operation mode and the CSF characteristics, which agrees with the empirical data.

Thus, an approach based on the use of stochastic methods and data from empirical studies makes it possible to obtain an estimate of the efficiency of dust collectors.

\section{References}

[1] V.N. Azarov, D. P. Borovkov, A. M. Red, "Application of swirling flows in aspiration systems, International Review of Mechanical Engineering, Vol. 8, Issue 4, pp. 750-753, IF 6,46, 2014.

[2] V.N. Azarov, D.V. Lukanin, D.P. Borovkov, A.M. Redhwan, "Experimental study of secondary swirling flow influence on flows 
[8] M. Newson-Smith, "Cement dust exposure and ventilatory function impairment: an exposure-response study," J. Occup. Environ. Med., Vol. 47, Iss. 1, pp. 1, 2005.

structure at separation chamber inlet of dust collector with counter 8, Issue 5, pp. 851-856, IF 6,46, 2014.

[3] V.N. Azarov, I. N. Logachev, K. I. Logachev, et al. "Methods of reducing the power requirements of ventilation systems Part 4," The oretical prerequisites with swirling air flows, Refractories and industrial Ceramies, Vol. 55, Issue 4, pp. 365-370, 2014.

[4] V.N. Azarov, N. V. Menzelitseva, et al. "Main Trends of Conditions Normalizing at Cement Manufacturing Plants," International Review of Civil Engineering, Vol. 6. N. 6, pp. 140-145, IF 6,46, 2016.

[5] V.N. Azarov, S.A. Koshkarev, "Evaluation of wet dust separator effectiveness in the dedusting of emissions from expanded clay kiln," Magazine of Civil Engineering, Vol. 54(2), pp. 18-32.

[6] V.N. Azarov, et al., "Aerodynamic characteristics of dust in the emissions into the atmosphere and working zone of construction enterprises," International Review of Civil Engineering, Vol. 7(5), pp. 132-136, 2016.

[7] V.N. Azarov, Koshkarev, S.A., D.V. Azarov, "The Decreasing Dust Emissions of Aspiration Schemes Applying a Fluidized Granular Particulate Material bed Separator at the Building Construction Factories," Procedia Engineering, Vol. 165, pp. 1070-1079, 2016.
[9] V.N. Azarov, M.V. Trokhimchuk, O.P. Sidelnikova, "Research of dust content in the earthworks working area," Procedia Engineering, Vol. 150, pp. 2008-2012, 2016.

[10] V.N. Azarov, N.S. Barikaeva, T. Solovyeva, "Monitoring of fine particulate air pollution as a factor in urban planning decisions," Procedia Engineering, Vol. 150, pp. 2001-2007, 2016.

[11] V.N. Azarov, M.V. Trohimchuk, K.A. Trohimchuk, "Empirical studies of the processes of dust distribution in the areas of construction dumps," News of higher education institutions, Geology and exploration, № 1, pp. 55-59, 2016.

[12] V.N. Azarov, Comprehensive assessment of the dust situation and development of measures to reduce dustiness of air environment of industrial enterprises, Dissertation, Rostov-Don, 2004.

[13] V.I. Tihonov, M.A. Mironov, Markov processes, Moscow, «Soviet radio», 1977, pp. 307-315.

[14] E.I. Boguslavsky, "Effectiveness of mass transfer in the centrifugal field of dust collecting apparatuses taking into account impact interactions of particles," News of higher education institutions. Construction, Vol. 5, pp 76-80, 1996. 